\title{
Bone remodelling considering drug effects on a damage mechanics model
}

\author{
M. E. Zeman ${ }^{1}$ J. M. García ${ }^{2}$, M. Cerrolaza ${ }^{1}$ \& M. Doblaré ${ }^{2}$ \\ I Bioengineering Centre, Faculty of Engineering, Central University of Venezuela \\ ${ }_{2}$ Structural Mechanics Division, Mechanical Eng. Dep. Centro Politecnico \\ Superior, University of Zaragoza, Spain
}

\begin{abstract}
It is well known that mechanical loads are one of the main factors that affect bone remodelling process. This phenomenon is widely applied to the study of prosthetic replacement, for example hip prosthesis. Elderly people are the most common patient to receive a hip replacement surgery.

Alendronate is a drug that is being used to increase bone mineral content in patients with poor bone quality.

This work uses a mechanical model based on damage mechanics, which considers bone's porosity as the damage variable, a mechanical stimulus associated thermodynamically to this variable, a damage criterion and an evolution damage law. Our approach simulates the degree of mineralization changes as well as the decrease of BMU activation frequency due to Alendronate doses. The biological changes generated by the drug directly affect the damage's evolution law. As a first approach the two biological parameters to be modeled are the surface of remodelling as well as the degree of mineralization (ash fraction) of the bone. Results show a good correlation with experimental data from alendronate's treatments for short term simulations (1-2 years).
\end{abstract}

\section{Introduction}

Cyclic loading is a key factor in bone remodelling. Many phenomenological remodelling models show this process very clearly, but loads are only one of the factors that are involved regarding remodelling. Biological processes in bone are affected of course by these loads but genetics as well as drugs or hormones have an important role to be considered as well.

Most of the patients that have to be under hip prosthesis surgery are elderly people who already have osteopenic or even osteoporotic bones and have to take drugs to increase their bone quality. Thus we incorporated a drug effect in a bone 


\section{6}

Simulations in Biomedicine $V$

remodelling theory. Moreover this new remodelling theory has been implemented in a 3D mechanical model using the finite element method.

In this work we developed a biological model that reflects the results of an osteoporosis treatment and coupled it to an existing bone remodelling model by Doblaré \& García [1] based on damage mechanics. Both models are coupled by variables affecting the biological behaviour as well as the flow law in the mechanical model, which shows the evolution of the damage, parameter considered to be the porosity.

\subsection{Alendronic Acid in BMU activity}

Alendronate (alendronic acid) is a bisphosphonate compound that has demonstrated biochemical stability and pharmacological activity as inhibitors of bone resorption and, thus, have an expanding role in the clinical management of patients with bone disease. Alendronate is used as an oral medication for both treatment and prevention of postmenopausal osteoporosis, corticosteroid-induced osteoporosis and the treatment of Paget's disease. As an example, women with postmenopausal osteoporosis, taken alendronate, have shown an increase in bone mineral density (BMD) and a reduction in fracture incidence, including at the hip. Bone mineral density can be directly related with the mechanical properties of bone, and the same amount of bone tissue having either a high or a low degree of mineralization will correspond to a higher or a lower BMD. This allows following these treatments by Dual X-ray Absoptiometry (DXA).

Studies performed by Porras et al. [2] show that after alendronate doses administration, the mechanism of action of alendronate is focused in areas of high physiological activity, where bone turnover is greatest. The drug acts by changing the bone remodelling process and different results have been associated with alendronate treatment as to mention among others: decreased remodelling space and increased mineralization of the bone.

Since remodelling is a process involving the action of osteoclast cells removing bone, coupled with osteoblast cells forming bone, they are considered to work together as Basic Multicellular Units (BMUs). Each BMU resorbs a small portion of bone followed by the formation of new bone. In osteoporosis, there is a negative unbalanced coupling between bone resorption and bone formation, resulting in bone loss. This loss is accelerated by increase in the activation frequency of new remodelling units (changes in bone turnover can be represented by changes in activation frequency of an BMU); this is induced by menopause and persists until the end of life.

As bone resorption and formation are coupled, we can expect that any influence in one component of turnover is followed by a response in its complementary process. Then we could consider that inhibition of bone resoption will bring an inhibition of bone formation. This effect of bisphosphonate administration has been studied by based on histomorphometric studies showing that the activation frequency of the BMUs, is reduced by an average of $87 \%$ for $10 \mathrm{mg} / \mathrm{day}$ alendronate doses. Parfitt et al. [3] have explained that the activation frequency, as a measure of the birth rate of cross-sectional cycles of remodelling is a 
valuable measure of the magnitude of the remodelling space. In our model the effect of alendronate is simulated by a factor that reduces the remodelling surface in the same proportion that the one is clinically observed in activation frequency Another aspect to be considered is the mean degree of mineralization of bone. In adults, this variable depends on the rate of remodelling. In other words, the biological determinant of mineralization is the rate of turnover or related activation frequency.

In the case of osteoporosis an increase of activation frequency brings to a shortened lifespan of the BMUs. Consequently, the new formed bone will not have enough time to reach its full degree of mineralization before being prematurely reabsorbed by osteoclast starting a new remodelling sequence. Data obtained from patients with osteoporosis indicate that the average value for ash fraction (ash mass / bone mass) was variable, but high mineralization observed after drug treatment was attributed to longer periods of secondary mineralization [4].

This brings us to define two different types of mineralization processes. First the initial deposition of mineral in new formed bone, which occurs very quickly and is called primary mineralization; is taken to be the period of time that will require a bone sample to reach up to $65 \%-75 \%$ of its maximum value [5]. And the secondary mineralization period, during which the mineral continues to accumulate at an exponentially decreasing rate, until the bone gets mature and reaches its maximum ash value.

It is hypothesized in Meunier and Boivin [4] that a reduced turnover increases the lifespan of BMUs and leads to more mature bone in which at least a normal level in their degree of mineralization can be reached. This result has been associated with alendronate treatment and is simulated in this model by calculating the variations of the ash fraction and by incrementing that parameter on a daily basis.

\section{Methods}

Initial homogeneous apparent density related to each integration point is loaded from a computer file. The biological model introduces the ash fraction as a new variable that reflects the degree of mineralization of the apparent density measurements. This is implemented using a logarithmic approximation; see eqn (1) relating the apparent density $\left(\rho_{a}\right)$ and its associated ash fraction $\left(a \mathrm{sh}_{0}\right)$ as an initial condition. Data to build the equation was taken from experimental results by Keller [6].

$$
a s h_{0}=0.2264 * \ln \left(\rho_{a}\right)+0.1772
$$

A total mineralised bone density $(\hat{\rho})$ is then calculated using a linear relation with values of ash fraction observed in humans and ranging from osteoid to cortical bone table (1). 
Table 1: Range of values for dry tissue density and ash fractions used in this model.

\begin{tabular}{|l|c|c|}
\hline & $\begin{array}{c}\hat{\rho} \\
(\mathrm{g} / \mathrm{cc})\end{array}$ & $\begin{array}{l}\text { Ash } \\
\text { fraction }\end{array}$ \\
\hline Osteoid & 1.5 & 0 \\
\hline Cortical bone & 2.04 & 0.70 \\
\hline
\end{tabular}

Changes in ash fraction are calculated based on daily increments rates. As reported by Hernandez et al [7] the variations of ash is implemented with two curves, a linear one for the primary mineralization period, and a logarithmic one for the slower secondary mineralization period (in this model considered to be 3 years) Fig (1). For each increment, evaluating the variation of the ash fraction, the program recalculates the total density parameter of the mechanical model.

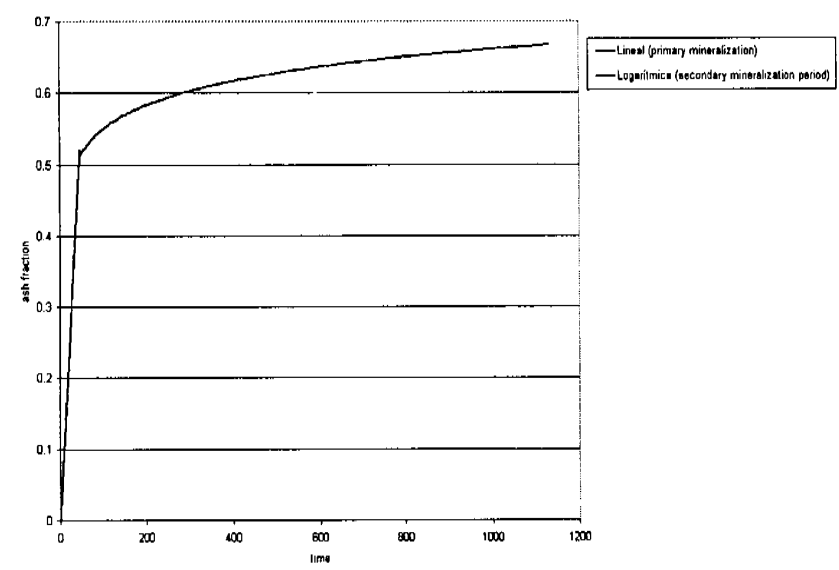

Figure 1: Curves representing the mineralization periods.

Regarding the remodelling surface changes, an expression used by Hernandez et al. [8] to represent the pharmacokinetics (distribution and elimination of alendronate in the bone) and pharmacodinamics (the relationship between drug concentration and physiological response) of alendronate was implemented, see eqn (2).

$$
S v_{\text {factor }}=a^{*}\left(1-m^{*} \ln (t+1)\right)
$$

where $\mathrm{Sv}_{\text {factor }}$ is the value to multiplied the remodelling surface $\mathrm{Sv}$ in normal conditions, $a$ represents the percent decrease in remodelling surface reflecting decreases in activation frequency generated by alendronate, in this case a decrease of $87 \%$ is used following clinical data; $m$ is a constant that is 
determined by fitting the equation to the clinical data, a value of $m=0.055$ was used to obtain results for weekly doses of alendronate $(70 \mathrm{mg} /$ week), $t$ represents time values multiplied by seven for weekly doses.

The biological changes are implemented using a dynamic feedback loop that is activated with a relationship between the surface for remodelling and the flow law, which determines the evolution of the damage variable in the mechanical model. Mineralization changes are calculated for every increment and stored for each integration point, see Figure 2.

A bending cyclic load of $40 \mathrm{~N}$ was applied to a bar as the cantilever case. Simulations of 18 months were performed and results were followed at certain control nodes.

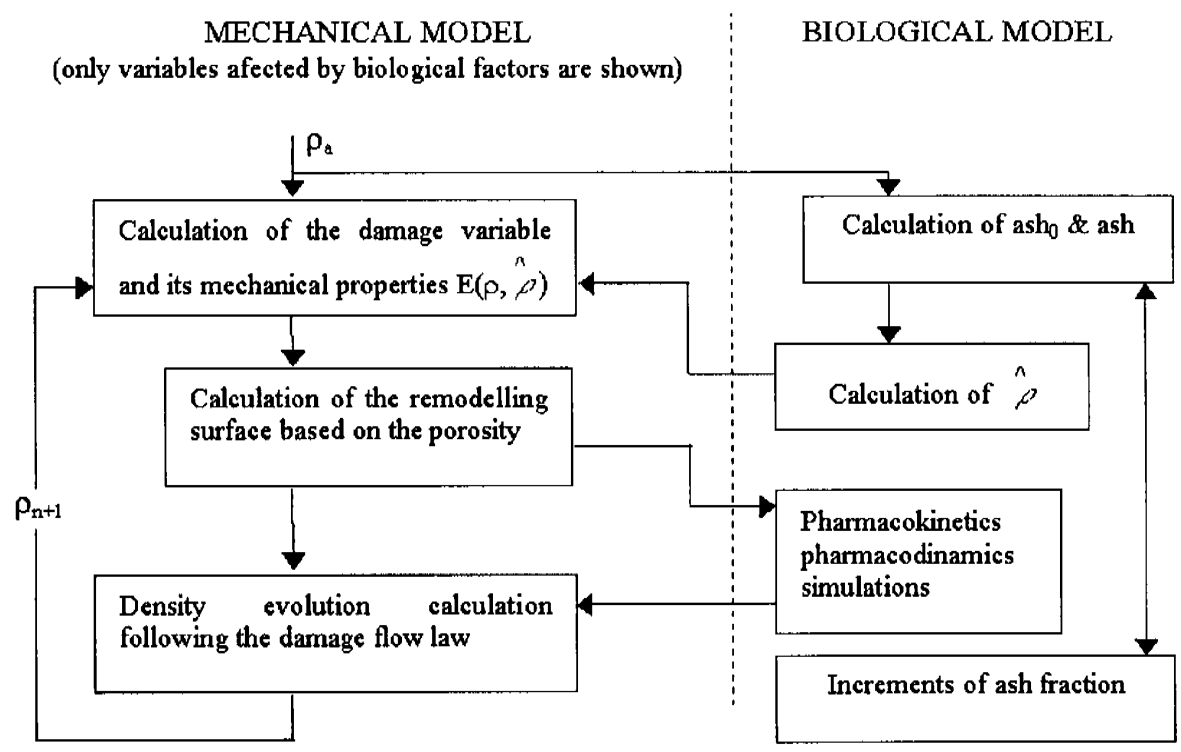

Figure 2: Diagram of the two models coupling.

\section{Results}

An average increase of $5.6 \%$ in the density was obtained at the end of the first year simulated and a $6.6 \%$ for the end of the 18 month simulation, as shown in Fig (3).

Variations simulating the periodic decrease of the activation frequency are reflected in the remodelling surface and are depicted in Figure (4). They offered a good correspondence with pharmacokinetis and pharmacodinamics of alendronate. 
Sensibility analyses showed that for variations in the parameters involved in the mineralization equations, the response of the output variable, density, is highly dependant. Less correlation was found between the variation of the remodelling surface and the density increase.

\section{Discussion}

Although the model generates a discrepant result for the first period of treatment simulation (too high increase), the response tends to be steady and logical for the rest of the simulated period. This behaviour should be corrected but it is important to mention that since changes in bone mineral density caused by alendronate are almost imperceptible before the first year of treatment, this result can be consider accurate as a preliminary result taking into account only the values obtained at the end of the period simulated. Results for those periods are comparable with the ones finded for clinical studies in the literature. For weekly treatment: increase in BMD of $5.1 \pm 0.3 \%$ after one year and $6.8 \pm 0.4$ after 2 years [7]. Long term simulations have to be performed in order to assess the stability of the model.

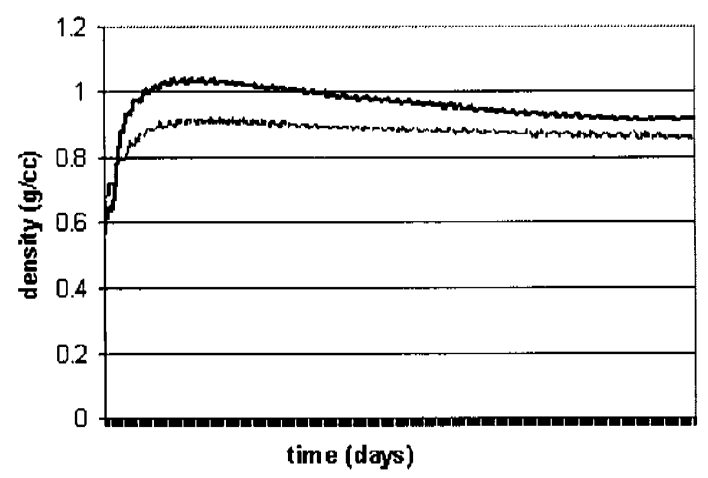

Figure 3: Results of the density variation in time. Upper line depicts predictions obtained for the alendronate simulation. Lower line is the control reference of the mechanical model.

A relevant comment should be made about the nature of the variables used in this model. The mechanical model makes an approach to the remodelling phenomena from the macroscopic point of view of the continuum, although microstructure variables as porosity are taken into account. The biological phenomena modelled in this approach are the result of cellular behaviour that can be measurable and represented in the macro model in a consistent way. This discussion was considered to couple both models and to develop a platform that will allow the interaction of more biological parameters in the remodelling process.

Another aspect about the coupling is that the effect of alendronate treatment is not considered as an additional stimulus entry, but as a parameter affecting the temporary evolution of the apparent density. 


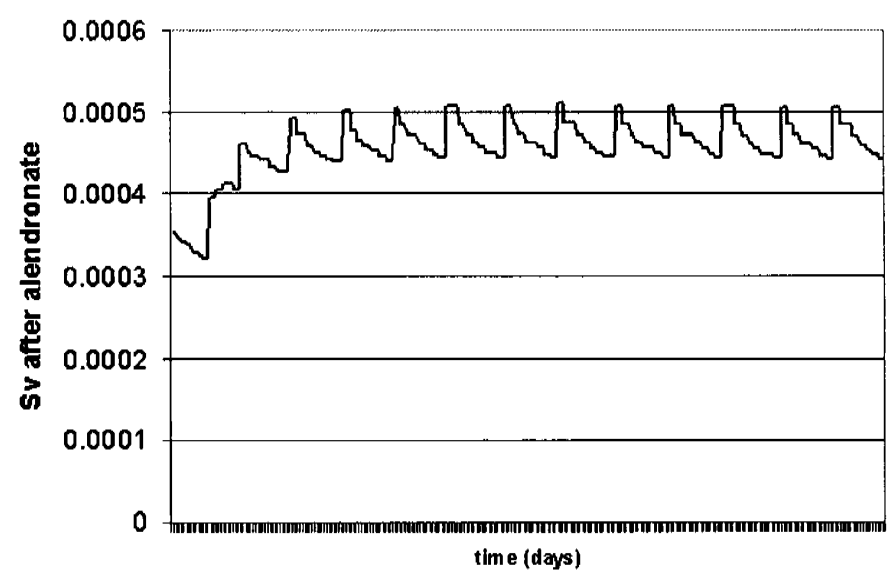

Figure 4: A detail of the surface of remodelling variation in time, after alendronate simulation is included for weekly doses.

These preliminary results show a good performance between the two models, this encourage us to keep incorporating more biological parameters in order to get a closer approach to the relation between mechanical and biological functions in bone remodelling.

\section{References}

[1] Doblaré, M., García J.M., Anisotropic bone remodelling model based on a continuum damage-repair theory. $J$ of Biomechanics, 35, pp. 1-17, 2002

[2] Porras A., Holland S. and Gertz B., Pharmacokinetics of Alendronate. Clinical Pharmacokinetics. vol. 36(5):315-328, 1999.

[3] Parfitt A., Mundy G., Roodman G., Hughes D. and Boyce B., A new model for the regulation of bone resorption, with particular reference to the effect of bisphosphonates. J. of Bone and Mineral Research. vol. 11(2):150-157, 1996.

[4] Meunier P.and Boivin G., Bone mineral density reflects bone mass but also the degree of mineralization of bone: therapeutic implications. Bone. vol. 21(5):373-377, 1997

[5] Roberts, E., Bone tissue interface. J of dental education. vol. 52(12), pp. 804809,1988

[6] Keller, T., Predicting the compressive mechanical behavior of bone. $J$ of Biomechanics. vol. 27(9):1159-1168, 1994

[7] Hernandez, C., Beaupré, G., Marcus, R., Carter, D., A theoretical analysis of the contributions of remodeling space, mineralization and bone balance to changes in bone mineral density during alendronate treatment. Bone, 29(6), pp. 511-516, 2001.

[8] Hernandez, C., Beaupré, G., Marcus, R., Carter, D., Long-term predictions of the therapeutic equivalence of daily and less than daily alendronate dosing. $J$ of Bone \& Min Research, 17(9), pp. 1662-1666, 2002. 
